\title{
三氯异氰尿酸促进下腙的氯化反应研究
}

\author{
宗吴中 \\ 胡雨来* \\ 王小平 \\ 刘佳欣 \\ 黄丹凤 \\ 王克虎
}

(西北师范大学化学化工学院 兰州 730070)

\begin{abstract}
摘要 以三氯异㲵尿酸为氯源，探索了腙和三氟乙醛芳腙的氯化反应，高产率地得到了一系列氯代芳腙和三氟乙酰氯 芳腙化合物。该方法具有反应条件温和，反应时间短，易于操作，氯源稳定、价格低廉等特点，提供了一种高效合成氯 代芳腙及三氟乙酰氯芳腙的新方法.

关键词 三氯异氰尿酸; 氯化反应; 氯代芳腙; 三氟乙酰氯芳腙; 合成
\end{abstract}

\section{Study on the Chlorination Reaction of Hydrazones by Using of Trichloroisocyanuric Acid as Chloride Source}

\author{
Zong, Wuzhong \\ $\mathrm{Hu}$, Yulai* \\ Wang, Xiaoping \\ Liu, Jiaxin \\ Huang, Danfeng \\ Wang, Kehu \\ (College of Chemistry and Chemical Engineering, Northwest Normal University, Lanzhou 730070)
}

\begin{abstract}
The chlorination reaction of hydrazones with $N$-aryltrifluoroacetohydrazones by using trichloroisocyanuric acid as chlorine source was investigated, and a series of $N$-arylhydrazonyl chlorides and $N$-aryltrifluoroacetohydrazonyl chlorides were obtained in high yields. This method had advatages of easy operation, short reaction time, mild conditions and the use of stable and cheap chlorine source, which provides an efficiant method for the synthesis of $N$-arylhydrazonyl chlorides and $\mathrm{N}$-aryltrifluoroacetohydrazonyl chlorides.

Keywords trichloroisocyanuric acid; chlorination reaction; hydrazonyl chlorides; trifluoroacetohydrazonyl chlorides; synthesis
\end{abstract}

卤代腙在碱的作用下生成 1,3-偶极子腈亚胺 ${ }^{[1]}$, 可 与碳碳双键、碳碳参键、碳氧双键、碳氮双键以及碳氮

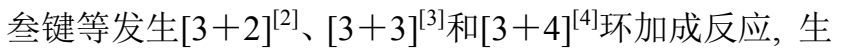
成各种含氮的五元、六元及七元杂环化合物等. 此外, 卤代腙也能与有机金属试剂发生偶联反应生成结构复 杂的酮腙类化合物，而这些酮腙又是合成具有生物活性 的杂环化合物的重要原料 ${ }^{[5]}$. 因此, 卤代腙在杂环化合 物的合成中占有重要地位. 目前, 卤代腙的合成通常是 通过腙、酰腙、酰肼或重氮盐的卤化反应而获得 ${ }^{[5]}$. 在 氯代芳腙的制备中，常用的氯化试剂有氯气、五氯化磷、 二氯亚砜、四氯化碳、 $N$-氯代琥珀酰亚胺 $(\mathrm{NCS})^{[1 \mathrm{c}]}$ 等. 其 中, $N$-氯代琥珀酰亚胺是较好的氯源，其他氯源都存在 反应选择性差，副反应多，反应条件苛刻等缺陷 ${ }^{[6]}$. 因 此，探索一种更为简便、高效的合成氯代芳腙的方法仍

\section{然十分必要.}

三氯异氰尿酸(简称 TCCA)已被广泛应用于饮用水 处理、游泳池消毒、食品防腐和羊毛的非缩水处理等家 庭生活和工业生产中 ${ }^{[7]}$ ，具有安全性好、易于操作、耐 储存及价格低廉等优点. 在全球范围内, 三氯异氰尿酸 的生产规模年产可达十万吨左右. 在有机合成中，三氯 异氰尿酸主要作为高效、稳定的氯化试剂 ${ }^{[8]}$ 和氧化剂 ${ }^{[9]}$. 在三氯异氰尿酸作为氯源的氯化反应中，其分子中的高 氯含量(45.8\%)使反应具有很好的原子经济性; 副产物 异氰尿酸易于分离, 又可用于重新生产三氯异氰尿酸, 符合绿色化学的原理. 因此，三氯异氰尿酸在有机合成 中有广泛的应用。本文报道以三氯异氰尿酸为固体氯 源，通过腙与三氯异氰尿酸的反应合成氯代腙的新方 法. 由于含三氟甲基的有机物在医药、农药和材料科学

* Corresponding author. E-mail: huyl@nwnu.edu.cn

Received November 30, 2018; revised January 17, 2019; published online January 31, 2019.

Project supported by the National Natural Science Foundation of China (No. 21662030).

国家自然科学基金(No. 21662030)资助项目. 
领域中有广泛应用, 通过三氟乙醛芳腙与三氯异氰尿酸 的反应也合成了三氟乙酰氯芳腙化合物, 期望为合成含 三氟甲基的化合物提供一种三氟甲基合成砌块.

\section{1 结果与讨论}

\subsection{TCCA 促进下腙的氯化反应研究}

首先，尝试了苯甲醛苯腙(1a) (1.0 equiv.)、TCCA 2 (0.4 equiv.) 与二甲硫醚(3) (1.2 equiv.) 的反应 ${ }^{[6]}$. 结果发 现, 在二氯甲烷溶剂中, $0{ }^{\circ} \mathrm{C}$ 条件下, 反应 $170 \mathrm{~min}$ 后以 $51 \%$ 的收率得到了目标产物 $4 \mathbf{a}$. 为了进一步提高目标产 物 $4 \mathbf{a}$ 的产率, 该反应的物料比进行了优化(表 1, Entries $1 \sim 3)$. 研究表明, 苯甲醛苯腙(1a)、TCCA (2)和二甲硫 醚(3)的物质的量的比为 $1: 0.8: 2.4$ 时, 目标产物 $4 \mathbf{a}$ 的 产率可达到 $75 \%$, 且反应时间大大缩短, 仅在 $50 \mathrm{~min}$ 内 即可完成(表 1, Entry 3). 然后, 在此条件下对该反应的 温度进行了考察 (表 1 , Entries 4 6). 当降低反应温度为 $-15{ }^{\circ} \mathrm{C}$ 时, 对目标产物 $\mathbf{4 a}$ 的产率影响不大, 但是反应 时间增加; 当提高反应温度到室温时, 反应时间虽然缩 短为 $30 \mathrm{~min}$, 但是目标产物 $\mathbf{4 a}$ 的产率也没有提高. 值得 高兴的是: 当反应在 $0 \sim 25{ }^{\circ} \mathrm{C}$ 进行时, 目标产物 $4 \mathrm{a}$ 的 产率可以提高到 $82 \%$, 反应仅需 40 min (表 1, Entry 6). 最后, 考察了溶剂对反应的影响(表 1, Entries 7〜12). 结果发现, 氯代溶剂 1,2-二氯乙烷和三氯甲烷对该反应 也是有利的(表 1, Entries 7〜8); 其他溶剂如乙腈、乙酸 乙酯、四氢呋喃、丙酮等对该反应不利. 为了探索该氯
化反应的反应机理，做了一个对照试验，即在不添加二 甲硫醚 3 的情况下，尝试了苯甲醛苯腙 $1 \mathbf{a}$ 与 TCCA 2 的 反应, 没有得到目标产物 4a (表 1, Entry 13), 说明在 TCCA 促进下芳腙的氯代反应中，二甲硫醚是必不可少 的. 因为在该反应中, TCCA 首先与二甲硫醚形成锍盐 ${ }^{[6]}$ 之后才能与苯腙发生氯代反应(详见第 1.3 节反应机理 部分). 综上所述, 合成目标产物 $\mathbf{4 a}$ 的最佳反应条件为: 苯甲醛苯腙 $(1 \mathrm{a}) 、 \operatorname{TCCA}(2)$ 和二甲硫醚 $(3)$ 的物质的量的 比为 $1: 0.8: 2.4$, 溶剂为二氯甲烷, 反应温度为 $0 \sim$ $25{ }^{\circ} \mathrm{C}$, 反应时间为 $40 \mathrm{~min}$.

为了探索该反应的适应性, 在上述最佳反应条件 下，对各种腙的氯化反应情况进行了考察，结果列于表 2. 首先对各种取代的苯甲醛与苯肼形成的苯腙进行了 拓展，发现苯甲醛的苯环上的取代基的电子性质对该反 应的影响不大，不管取代基是供电子的还是吸电子的， 所得氯代芳腙的产率都在 $62 \%$ 以上 $(4 \mathrm{~b} \sim 4 \mathrm{j})$. 但是取代 基在苯环上的位置对反应有较大影响. 例如，当 4-甲基 苯甲醛和 3-甲基苯甲醛形成的苯腙反应时，所得氯代芳 腙 $4 b$ 和 $4 c$ 的产率分别为 $80 \%$ 和 79\%，而 2-甲基苯甲醛 形成的苯腙却不发生反应，没有得到目标产物; 当 4-氯 苯甲醛和 3-氯苯甲醛形成的苯腙反应时，所得氯代芳腙 4f 和 $4 \mathrm{~g}$ 的产率分别为 $83 \%$ 和 $77 \%$, 但是 2-氯苯甲醛形 成的苯腙反应时, $4 \mathrm{~h}$ 的产率降低为 $64 \%$. 当 2-䒺甲醛和 2-呋喃甲醛形成的苯腙作为底物反应时, $4 k$ 和 41 的产率 分别为 $78 \%$ 和 $74 \%$. 对于苯甲醛和各种取代的苯肼形

表 1 氯化反应的条件优化 ${ }^{a}$

Table 1 Optimization of chlorination reaction conditions

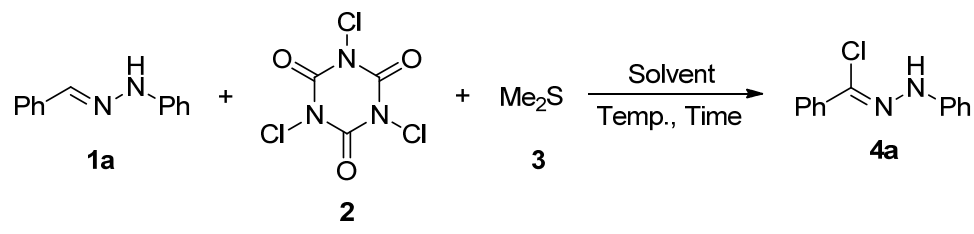

\begin{tabular}{|c|c|c|c|c|c|}
\hline Entry & Molar ratio of $1 \mathbf{a} / \mathbf{2} / \mathbf{3}$ & Solvent & Temp. $/{ }^{\circ} \mathrm{C}$ & Time/min & Isolated yield $/ \%$ of $\mathbf{4 a}$ \\
\hline 1 & $1.0 / 0.4 / 1.2$ & $\mathrm{CH}_{2} \mathrm{Cl}_{2}$ & 0 & 170 & $51\left(80^{b}\right)$ \\
\hline 2 & $1.0 / 0.6 / 1.8$ & $\mathrm{CH}_{2} \mathrm{Cl}_{2}$ & 0 & 170 & 77 \\
\hline 3 & $1.0 / 0.8 / 2.4$ & $\mathrm{CH}_{2} \mathrm{Cl}_{2}$ & 0 & 50 & 75 \\
\hline 4 & $1.0 / 0.8 / 2.4$ & $\mathrm{CH}_{2} \mathrm{Cl}_{2}$ & $0 \sim-15$ & 80 & 73 \\
\hline 5 & $1.0 / 0.8 / 2.4$ & $\mathrm{CH}_{2} \mathrm{Cl}_{2}$ & $0 \sim 25$ & 30 & 74 \\
\hline 6 & $1.0 / 0.8 / 2.4$ & $\mathrm{CH}_{2} \mathrm{Cl}_{2}$ & $0 \sim 25$ & 40 & 82 \\
\hline 7 & $1.0 / 0.8 / 2.4$ & DCE & $0 \sim 25$ & 40 & 78 \\
\hline 8 & $1.0 / 0.8 / 2.4$ & $\mathrm{CHCl}_{3}$ & $0 \sim 25$ & 40 & 80 \\
\hline 9 & $1.0 / 0.8 / 2.4$ & THF & $0 \sim 25$ & 40 & Trace \\
\hline 10 & $1.0 / 0.8 / 2.4$ & $\mathrm{CH}_{3} \mathrm{CN}$ & $0 \sim 25$ & 40 & $38\left(77^{c}\right)$ \\
\hline 11 & $1.0 / 0.8 / 2.4$ & Acetone & $0 \sim 25$ & 40 & Trace \\
\hline 12 & $1.0 / 0.8 / 2.4$ & Ethyl acetate & $0 \sim 25$ & 40 & $14\left(72^{d}\right)$ \\
\hline 13 & $1.0 / 0.8 / 0$ & $\mathrm{CH}_{2} \mathrm{Cl}_{2}$ & $0 \sim 25$ & 40 & 0 \\
\hline
\end{tabular}

${ }^{a}$ All reactions were carried out by using $0.5 \mathrm{mmol}$ of $\mathbf{1 a}$, and $4 \mathrm{~mL}$ of solvent; ${ }^{b}$ Based on $64 \%$ conversion of $1 \mathrm{a} ;{ }^{c}$ Based on $50 \%$ conversion of $1 \mathrm{a} ;{ }^{d}$ Based on $19 \%$ conversion of 1a. 
成的芳腙, 反应都能顺利进行, 并以良好产率得到目标 产物 $(4 \mathrm{~m} \sim 4 p)$. 对于各种取代的苯甲醛和取代的苯肼形 成的芳腙来说, 反应也能发生, 但是产物产率有所降低 $(4 q \sim 4 t)$. 最后, 对脂肪族醛与苯肼形成的苯腙也尝试 了该反应. 例如当肉桂醛和苯丙醛形成的苯腙反应时, $4 \mathbf{u}$ 和 $4 v$ 的产率分别为 74\% 和 76\%. 环戊基甲醛和环己 基甲醛形成的苯腙也能顺利进行反应, 所得产物 $4 \mathrm{w}$ 和 4x 的产率依次为 $58 \%$ 和 $64 \%$. 然而, 开链脂肪醛, 如 乙醛和 2,2-二甲基丙醛形成的苯腙作为底物时, 反应体 系复杂, 没有得到相应的氯代腙. 此外, 乙肼、叔丁基肼 和环己基肼分别与苯甲醛形成的苯甲醛乙腙、苯甲醛叔 丁腙和苯甲醛环己基腙也尝试了该氯化反应, 遗憾的是 反应都没有发生

\subsection{TCCA 促进下三氟乙醛芳腙的氯化反应研究}

在上述研究结果及文献报道基础上 ${ }^{[10]}$, 我们又探 索了 TCCA 促进下三氟乙醛芳腙的氯化反应, 得到了一 些三氟乙酰氯芳腙化合物. 从表 3 可以看出, 不论三氟 乙醛芳腙的苯环上连有吸电子基团还是供电子基团，氯 化反应都能顺利进行, 并且以较好产率得到了目标化合 物 6 , 但是苯环上连有吸电子基的三氟乙醛芳腙比连有 供电子基的反应产率高. 例如苯环上连有氟原子和硝基 的三氟乙醛芳腙作为底物反应时, 所得产物 $6 \mathrm{~g}$ 和 $6 \mathrm{~h}$ 的 产率分别可达 $86 \%$ 和 $82 \%$; 而苯环上连有甲基和甲氧基 的三氟乙醛芳腙作为底物反应时，所得产物 $6 \mathrm{~b}$ 和 $6 \mathrm{c}$ 的 产率分别只有 $71 \%$ 和 $65 \%$.

表 2 腙的适用范围 ${ }^{a}$

Table 2 Substrate scope of hydrazones

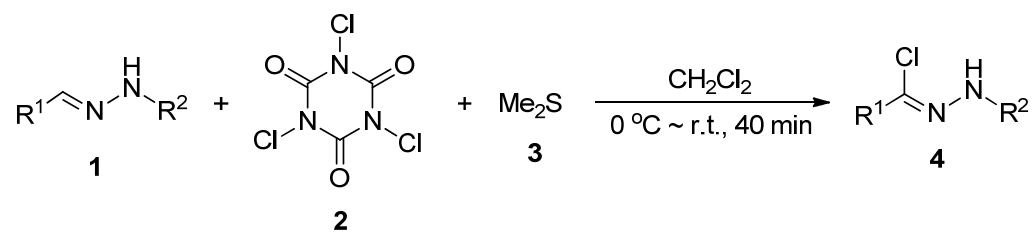<smiles>COc1ccc(C(Cl)=NNc2ccccc2)cc1</smiles>

${ }^{a}$ All reactions were carried out by using $0.5 \mathrm{mmol}$ of $\mathbf{1}, 0.4 \mathrm{mmol}$ of TCCA, $1.2 \mathrm{mmol}$ of $\mathrm{Me}_{2} \mathrm{~S}, 4 \mathrm{~mL}$ of $\mathrm{CH}_{2} \mathrm{Cl}_{2}$ as solvent, $0{ }^{\circ} \mathrm{C} \sim \mathrm{r}, \mathrm{t}$., $40 \mathrm{~min}$. 
表 3 三氟乙醛芳腙的适用范围 ${ }^{a}$

Table 3 Substrate scope of $N$-aryltrifluoroacetohydrazones

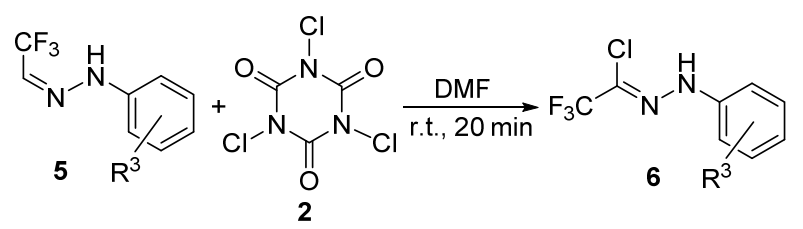<smiles>COc1ccc(NN=C(Cl)C(F)(F)F)cc1</smiles>

$\bar{a}$ All reactions were carried out by using $0.5 \mathrm{mmol}$ of $5,0.2 \mathrm{mmol}$ of TCCA, 3 $\mathrm{mL}$ of DMF as solvent, room temperature, $20 \mathrm{~min}$.

\section{3 反应机理探讨}

在文献报道 ${ }^{[6,11]}$ 和实验结果基础上, 我们认为
TCCA 促进下芳腙的氯化反应的反应机理如 Scheme 1 所示. 以苯甲醛苯腙 $(\mathbf{1 a})$ 的反应为例. 首先, TCCA (2)与 二甲硫醚 $(3)$ 作用生成 TCCA 的锍盐 7 , 锍盐 7 与苯甲醛 苯腙(1a)发生反应生成 TCCA 的锍盐 8 和苯甲醛苯腙的 锍盐 $9.7 C C A$ 的锍盐 8 继续与苯甲醛苯腙 $(\mathbf{1 a})$ 反应生成 锍盐 9 , 同时锍盐 8 变成锍盐 $\mathbf{1 0}$, 锍盐 10 再与苯甲醛苯 腙(1a)反应生成锍盐 9 和异氭尿酸 11. 然后, 生成的苯 甲醛苯腙的锍盐 9 脱去二甲硫醚(3)变成茮基碳正离子 12 , 氯负离子与此碳正离子结合生成偶氮化合物 13,13 再经过互变异构生成苯甲酰氯苯腙 4a.

\section{2 结论}

综上所述，本文以安全、高效的三氯异氰尿酸作为 固体氯源，实现了芳腙和三氟乙醛芳腙的氯化反应，以 较好产率得到了一系列氯代芳腙和三氟乙酰氯芳腙化 合物. 该方法与文献报道 ${ }^{[6,10]}$ 的方法相比，具有反应条 件温和、反应时间短、易于操作、氯源稳定、价格低廉 等特点, 提供了一种合成氯代芳腙及三氟乙酰氯芳腙的 新方法. 同时, 该方法也扩大了三氯异氰尿酸作为氯化 试剂在有机合成中的应用范围.

\section{3 实验部分}

\section{1 仪器与试剂}

NMR 用 BRUKER PT jxf790425AM $400 \mathrm{MHz}$ 或 Agilent $\mathrm{DD}_{2} 600 \mathrm{MHz}$ 型核磁共振仪测定, 以氝代氯仿 作为溶剂, TMS 为内标; 高分辨质谱用 Bruker APEXII 傅里叶变换离子回旋共振质谱仪测定, ESI 源; 质谱用 Thermo Trace-DSQ 气相色谱质谱联用仪测定, EI 源; 熔 点测定用显微熔点测定仪测定，温度未较正.

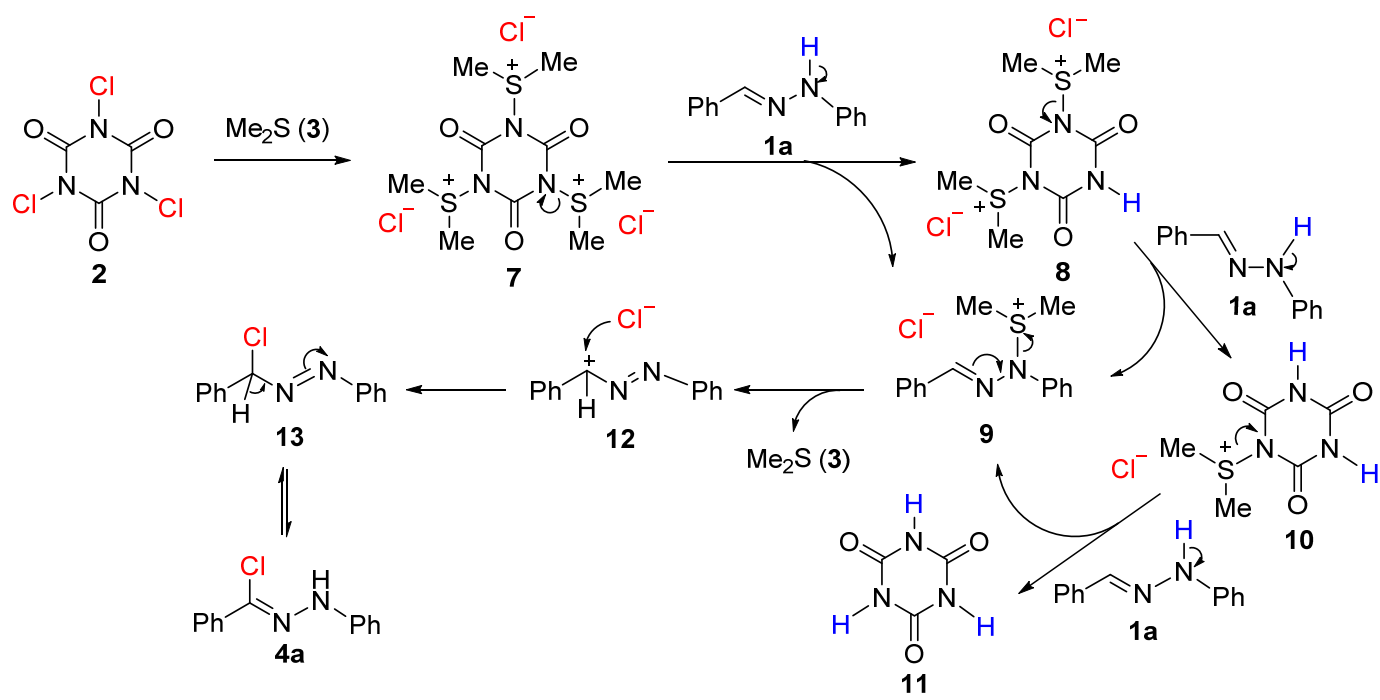

图式 1 可能的反应机理

Scheme 1 Proposed mechanism 
实验中所用起始原料化合物 $\mathbf{1 a} \sim 1 \mathrm{x}$ 均是根据文献 自制 ${ }^{[12]}, \mathbf{5 a} \sim 5 \mathrm{~h}$ 均是根据文献自制 ${ }^{[13]}$; 所用试剂均是国 产分析纯级试剂.

\section{2 实验方法}

\section{2 .1 化合物 $\mathbf{4}$ 的合成}

在 $25 \mathrm{~mL}$ 的圆底烧瓶中, 冰浴下依次加入 TCCA $(0.4 \mathrm{mmol}, 93 \mathrm{mg})$ 、二氯甲烷 $(2 \mathrm{~mL})$ 、二甲硫醚 $(3)(1.2$ $\mathrm{mmol}, 74 \mathrm{mg}$ )并搅拌 $20 \mathrm{~min}$, 出现大量白色乳浊物. 然 后将苯甲酫苯腙 $(1 \mathbf{a})(0.5 \mathrm{mmol}, 98 \mathrm{mg})$ 溶于二氯甲烷 $(2$ $\mathrm{mL}$ ) 并加入到上述混合物中, 摚拌反应 $5 \mathrm{~min}$ 后, 再将圆 底烧瓶移至室温下反应 $15 \mathrm{~min}$, 薄层色谱(TLC)监测反 应完全. 向反应体系中加蒸馏水 $(10 \mathrm{~mL})$ 淬灭反应, 用乙 酸乙酯萃取 $(10 \mathrm{~mL} \times 3)$, 分出有机相, 再用饱和氯化钠 水溶液 $(10 \mathrm{~mL})$ 洗涤, 无水硫酸镁干燥, 抽滤, 减压除去 溶剂. 残留物进行柱层析 $[V$ (石油醚) $: V($ 乙酸乙酯 $)=$ $30: 1$ ]分离得苯甲酰氯苯腙 $(\mathbf{4 a})$ : 白色晶体, $94 \mathrm{mg}$, 产 率 $82 \%$. m.p. $127 \sim 129{ }^{\circ} \mathrm{C}$ (文献值 ${ }^{[11]}$ : $127 \sim 128{ }^{\circ} \mathrm{C}$ ); ${ }^{1} \mathrm{HNMR}\left(\mathrm{CDCl}_{3}, 600 \mathrm{MHz}\right) \delta: 8.03(\mathrm{~s}, 1 \mathrm{H}), 7.92$ (d, $J=7.2$ $\mathrm{Hz}, 2 \mathrm{H}), 7.29 \sim 7.41(\mathrm{~m}, 5 \mathrm{H}), 7.17(\mathrm{~d}, J=7.2 \mathrm{~Hz}, 2 \mathrm{H})$, $6.94(\mathrm{t}, J=6.0 \mathrm{~Hz}, 1 \mathrm{H}) ;{ }^{13} \mathrm{C}$ NMR $\left(\mathrm{CDCl}_{3}, 150 \mathrm{MHz}\right) \delta$ : $143.5,134.6,129.5,129.3,128.5,126.5,124.8,121.3$, 113.6; HRMS (ESI) calcd for $\mathrm{C}_{13} \mathrm{H}_{11} \mathrm{ClN}_{2} \mathrm{Na}[\mathrm{M}+\mathrm{Na}]^{+}$ 253.0503, found 253.0502.

化合物 $\mathbf{4 b} \sim \mathbf{4 x}$ 的合成方法同 $\mathbf{4 a}$.

(4-甲基苯基)甲酰氯苯腙 $(\mathbf{4 b})$ : 白色晶体, $98 \mathrm{mg}$, 产 率 $80 \%$. m.p. $134 \sim 136{ }^{\circ} \mathrm{C}$ (文献值 ${ }^{[14]}$ : $\left.133 \sim 134{ }^{\circ} \mathrm{C}\right) ;{ }^{1} \mathrm{H}$ NMR $\left(\mathrm{CDCl}_{3}, 600 \mathrm{MHz}\right) \delta: 7.98(\mathrm{~s}, 1 \mathrm{H}), 7.80(\mathrm{~d}, J=7.8$ $\mathrm{Hz}, 2 \mathrm{H}), 7.30$ (t, $J=8.4 \mathrm{~Hz}, 2 \mathrm{H}), 7.16 \sim 7.21(\mathrm{~m}, 4 \mathrm{H})$, $6.93(\mathrm{t}, J=7.2 \mathrm{~Hz}, 1 \mathrm{H}), 2.38(\mathrm{~s}, 3 \mathrm{H}) ;{ }^{13} \mathrm{C} \mathrm{NMR}\left(\mathrm{CDCl}_{3}\right.$, $150 \mathrm{MHz}) \delta: 143.6,139.5,131.9,129.5,129.3,126.5$, 125.1, 121.1, 113.5, 21.4; HRMS (ESI) calcd for $\mathrm{C}_{14} \mathrm{H}_{14} \mathrm{Cl}$ $\mathrm{N}_{2}[\mathrm{M}+\mathrm{H}]^{+}$245.0846, found 245.0838.

(3-甲基苯基)甲酰氯苯腙(4c): 白色晶体, $96 \mathrm{mg}$, 产 率 79\%. m.p. 73 75 ${ }^{\circ} \mathrm{C}$ (文献值 ${ }^{[2 e]}$ : $74 \sim 76{ }^{\circ} \mathrm{C}$ ); ${ }^{1} \mathrm{H}$ NMR $\left(\mathrm{CDCl}_{3}, 400 \mathrm{MHz}\right) \delta: 8.01(\mathrm{~s}, 1 \mathrm{H}), 7.71(\mathrm{~d}, J=6.8$ $\mathrm{Hz}, 2 \mathrm{H}), 7.26 \sim 7.32(\mathrm{~m}, 3 \mathrm{H}), 7.15 \sim 7.17(\mathrm{~m}, 3 \mathrm{H}), 6.93(\mathrm{t}$, $J=7.2 \mathrm{~Hz}, 1 \mathrm{H}), 2.40(\mathrm{~s}, 3 \mathrm{H}) ;{ }^{13} \mathrm{C} \mathrm{NMR}\left(\mathrm{CDCl}_{3}, 150 \mathrm{MHz}\right)$ $\delta: 143.6,138.2,134.6,130.2,129.5,128.4,127.1,125.1$, 123.9, 121.3, 113.6, 21.6; HRMS (ESI) calcd for $\mathrm{C}_{14} \mathrm{H}_{14} \mathrm{Cl}$ $\mathrm{N}_{2}[\mathrm{M}+\mathrm{H}]^{+}$245.0840, found 245.0833..

(4-甲氧基苯基)甲酰氯苯腙(4e)：白色晶体，80 mg, 产率 $62 \%$. m.p. $116 \sim 117{ }^{\circ} \mathrm{C}$ (文献值 ${ }^{[2]}$ : $116 \sim 118{ }^{\circ} \mathrm{C}$ ); ${ }^{1} \mathrm{H}$ NMR $\left(\mathrm{CDCl}_{3}, 600 \mathrm{MHz}\right) \delta: 7.93(\mathrm{~s}, 1 \mathrm{H}), 7.85(\mathrm{~d}, J=$ $9.0 \mathrm{~Hz}, 2 \mathrm{H}), 7.30$ (t, $J=7.2 \mathrm{~Hz}, 2 \mathrm{H}), 7.15(\mathrm{~d}, J=8.4,2 \mathrm{H})$, $6.91 \sim 6.93(\mathrm{~m}, 3 \mathrm{H}), 3.84(\mathrm{~s}, 3 \mathrm{H}) ;{ }^{13} \mathrm{C}$ NMR $\left(\mathrm{CDCl}_{3}, 150\right.$
MHz) $\delta: 160.7,143.7,129.5,128.1,127.3,124.9,121.0$, 113.9, 113.4, 55.6; HRMS (ESI) calcd for $\mathrm{C}_{14} \mathrm{H}_{14} \mathrm{ClN}_{2} \mathrm{O}$ $[\mathrm{M}+\mathrm{H}]^{+}$261.0795, found 261.0794.

(4-氯苯基)甲酰氯苯腙(4f): 黄色晶体，110 mg, 产 率 83\%. m.p. $143 \sim 145{ }^{\circ} \mathrm{C}$ (文献值 ${ }^{[2]}$ : $142 \sim 144{ }^{\circ} \mathrm{C}$ ); ${ }^{1} \mathrm{H}$ NMR $\left(\mathrm{CDCl}_{3}, 600 \mathrm{MHz}\right) \delta: 8.03$ (s, 1H), 7.84 (d, $J=9.0$ $\mathrm{Hz}, 2 \mathrm{H}), 7.37$ (d, $J=9.0 \mathrm{~Hz}, 2 \mathrm{H}), 7.31(\mathrm{t}, J=7.2 \mathrm{~Hz}, 2 \mathrm{H})$, $7.16(\mathrm{~d}, J=7.8 \mathrm{~Hz}, 2 \mathrm{H}), 6.95(\mathrm{t}, J=7.2 \mathrm{~Hz}, 1 \mathrm{H}) ;{ }^{13} \mathrm{C}$ NMR $\left(\mathrm{CDCl}_{3}, 150 \mathrm{MHz}\right) \delta: 143.3,135.3,133.2,129.6,128.8$, 127.7, 123.7, 121.6, 113.7; HRMS (ESI) calcd for $\mathrm{C}_{13} \mathrm{H}_{11^{-}}$ $\mathrm{Cl}_{2} \mathrm{~N}_{2}[\mathrm{M}+\mathrm{H}]^{+}$265.0299, found 265.0305.

(3-氯苯基)甲酰氯苯腙 $(\mathbf{4 g})$ : 粉红色晶体，102 $\mathrm{mg}$, 产率 77\%. m.p. 66 68 ${ }^{\circ} \mathrm{C}$ (文献值 ${ }^{[2 \mathrm{e}]}$ ：66 68 $\left.{ }^{\circ} \mathrm{C}\right) ;{ }^{1} \mathrm{H}$ NMR $\left(\mathrm{CDCl}_{3}, 400 \mathrm{MHz}\right) \delta: 8.06(\mathrm{~s}, 1 \mathrm{H}), 7.89(\mathrm{~s}, 1 \mathrm{H}), 7.78$ (t, $J=3.6 \mathrm{~Hz}, 1 \mathrm{H}), 7.30 \sim 7.34(\mathrm{~m}, 4 \mathrm{H}), 7.17(\mathrm{~d}, J=8.0$ $\mathrm{Hz}, 2 \mathrm{H}), 6.96(\mathrm{t}, J=7.2 \mathrm{~Hz}, 1 \mathrm{H}) ;{ }^{13} \mathrm{C}$ NMR $\left(\mathrm{CDCl}_{3}, 150\right.$ MHz) $\delta: 143.1,136.3,134.7,129.7,129.6,129.2,126.4$, 124.6, 123.1, 121.7, 113.7; HRMS (ESI) calcd for $\mathrm{C}_{13} \mathrm{H}_{10^{-}}$ $\mathrm{Cl}_{2} \mathrm{~N}_{2} \mathrm{Na}[\mathrm{M}+\mathrm{Na}]^{+}$287.0113, found 287.0118.

(2-氯苯基)甲酰氯苯腙 $(4 \mathbf{h})$ ：白色晶体, $84 \mathrm{mg}$, 产率 $64 \%$. m.p. $63 \sim 65{ }^{\circ} \mathrm{C}$ (文献值 ${ }^{[2]}$ : $62 \sim 64{ }^{\circ} \mathrm{C}$ ); ${ }^{1} \mathrm{H}$ NMR $\left(\mathrm{CDCl}_{3}, 600 \mathrm{MHz}\right) \delta: 8.05(\mathrm{~s}, 1 \mathrm{H}), 7.58 \sim 7.59(\mathrm{~m}, 1 \mathrm{H})$, $7.43 \sim 7.45(\mathrm{~m}, 1 \mathrm{H}), 7.27 \sim 7.31(\mathrm{~m}, 4 \mathrm{H}), 7.14(\mathrm{~d}, J=7.8$ $\mathrm{Hz}, 2 \mathrm{H}), 6.93(\mathrm{t}, J=7.2 \mathrm{~Hz}, 1 \mathrm{H}) ;{ }^{13} \mathrm{C}$ NMR $\left(\mathrm{CDCl}_{3}, 150\right.$ MHz) $\delta: 143.2,134.3,133.0,131.4,130.7,130.4,129.5$, $126.8,121.5,120.5,113.6$; HRMS (ESI) calcd for $\mathrm{C}_{13} \mathrm{H}_{11-}$ $\mathrm{Cl}_{2} \mathrm{~N}_{2}[\mathrm{M}+\mathrm{H}]^{+}$265.0299, found 265.0301.

(4-溴苯基)甲酰氯苯腙 $(4 \mathbf{i})$ : 黄色晶体，118 mg, 产 率 76\%. m.p. $147 \sim 148{ }^{\circ} \mathrm{C}$ (文献值 ${ }^{[2 \mathrm{e}]}$ : $146 \sim 148{ }^{\circ} \mathrm{C}$ ); ${ }^{1} \mathrm{H}$ NMR $\left(\mathrm{CDCl}_{3}, 600 \mathrm{MHz}\right) \delta: 8.03(\mathrm{~s}, 1 \mathrm{H}), 7.77$ (d, $J=8.4$ $\mathrm{Hz}, 2 \mathrm{H}), 7.52$ (d, $J=9.0 \mathrm{~Hz}, 2 \mathrm{H}), 7.31(\mathrm{t}, J=8.4 \mathrm{~Hz}, 2 \mathrm{H})$, $7.16(\mathrm{~d}, J=7.2 \mathrm{~Hz}, 2 \mathrm{H}), 6.95$ (t, $J=7.2 \mathrm{~Hz}, 1 \mathrm{H}) ;{ }^{13} \mathrm{C}$ NMR $\left(\mathrm{CDCl}_{3}, 150 \mathrm{MHz}\right) \delta$ : 143.2, 133.6, 131.7, 129.6, 127.9, 123.7, 123.5, 121.6, 113.6. HRMS (ESI) calcd for $\mathrm{C}_{13} \mathrm{H}_{10} \mathrm{ClN}_{2}[\mathrm{M}-\mathrm{Br}]^{+}$229.0527, found 229.0527.

(4-三氟甲基苯基)甲酰氯苯腙 ${ }^{[2 \mathrm{a}]}(\mathbf{4 j})$ ：黄色晶体, $120 \mathrm{mg}$, 产率 $80 \%$. m.p. $122 \sim 124{ }^{\circ} \mathrm{C} ;{ }^{1} \mathrm{H}$ NMR $\left(\mathrm{CDCl}_{3}\right.$, $600 \mathrm{MHz}) \delta: 8.13(\mathrm{~s}, 1 \mathrm{H}), 8.01(\mathrm{~d}, J=8.4 \mathrm{~Hz}, 2 \mathrm{H}), 7.64(\mathrm{~d}$, $J=8.4 \mathrm{~Hz}, 2 \mathrm{H}), 7.33(\mathrm{t}, J=8.4 \mathrm{~Hz}, 2 \mathrm{H}), 7.18(\mathrm{~d}, J=7.8$ $\mathrm{Hz}, 2 \mathrm{H}), 6.98(\mathrm{t}, J=7.2 \mathrm{~Hz}, 1 \mathrm{H}) ;{ }^{13} \mathrm{C}$ NMR $\left(\mathrm{CDCl}_{3}, 150\right.$ MHz) $\delta: 143.0,137.8,130.8\left(\mathrm{q}, J_{\mathrm{C}-\mathrm{F}}=32.3 \mathrm{~Hz}\right), 129.6$, $126.6,125.5\left(\mathrm{q}, J_{\mathrm{C}^{-} \mathrm{F}}=3.8 \mathrm{~Hz}\right), 124.1\left(\mathrm{q}, J_{\mathrm{C}-\mathrm{F}}=270.6 \mathrm{~Hz}\right)$, 123.1, 121.9, 113.8; ${ }^{19} \mathrm{~F}$ NMR ( $\left.\mathrm{MHz}, \mathrm{CDCl}_{3}\right) \delta: 63.1$.

2-菜甲酰氯苯腙 $(\mathbf{4 k})$ : 白色晶体, $109 \mathrm{mg}$, 产率 $78 \%$. m.p. $136 \sim 138{ }^{\circ} \mathrm{C}$ (文献值 ${ }^{[2 e]}: 136 \sim 138{ }^{\circ} \mathrm{C}$ ); ${ }^{1} \mathrm{H}$ 
NMR $\left(\mathrm{CDCl}_{3}, 600 \mathrm{MHz}\right) \delta: 8.25(\mathrm{~s}, 1 \mathrm{H}), 8.10 \sim 8.12(\mathrm{~m}$, $2 \mathrm{H}), 7.87 \sim 7.89(\mathrm{~m}, 1 \mathrm{H}), 7.80 \sim 7.82(\mathrm{~m}, 2 \mathrm{H}), 7.47 \sim 7.51$ (m, 2H), 7.32 (t, $J=8.4 \mathrm{~Hz}, 2 \mathrm{H}), 7.21(\mathrm{~d}, J=7.8 \mathrm{~Hz}, 2 \mathrm{H})$, $6.95(\mathrm{t}, J=7.2 \mathrm{~Hz}, 1 \mathrm{H}) ;{ }^{13} \mathrm{C} \mathrm{NMR}\left(\mathrm{CDCl}_{3}, 150 \mathrm{MHz}\right) \delta$ : $143.4,133.7,133.2,132.0,129.5,128.7,128.2,127.8$, $127.0,126.7,126.5,125.1,123.6,121.4,113.6$; HRMS (ESI) calcd for $\mathrm{C}_{17} \mathrm{H}_{13} \mathrm{ClN}_{2} \mathrm{Na}[\mathrm{M}+\mathrm{Na}]^{+} 303.0659$, found 303.0646 .

2-呋喃甲酰氯苯腙(4l): 棕褐色晶体, $81 \mathrm{mg}$, 产率 74\%. m.p. $78 \sim 80{ }^{\circ} \mathrm{C}\left(\right.$ 文献值 ${ }^{[2 \mathrm{e}]}$ : $\left.76 \sim 78{ }^{\circ} \mathrm{C}\right) ;{ }^{1} \mathrm{H} \mathrm{NMR}$ $\left(\mathrm{CDCl}_{3}, 600 \mathrm{MHz}\right) \delta: 7.92(\mathrm{~s}, 1 \mathrm{H}), 7.49(\mathrm{~m}, 1 \mathrm{H}), 7.30(\mathrm{t}$, $J=8.4 \mathrm{~Hz}, 2 \mathrm{H}), 7.15(\mathrm{~d}, J=7.8 \mathrm{~Hz}, 2 \mathrm{H}), 6.94(\mathrm{t}, J=7.2$ $\mathrm{Hz}, 1 \mathrm{H}), 6.74(\mathrm{~d}, J=2.4 \mathrm{~Hz}, 1 \mathrm{H}), 6.47 \sim 6.48(\mathrm{~s}, 1 \mathrm{H}) ;{ }^{13} \mathrm{C}$ $\mathrm{NMR}\left(\mathrm{CDCl}_{3}, 150 \mathrm{MHz}\right) \delta: 148.0,144.0,143.2,129.5$, 121.4, 116.1, 113.6, 111.8, 110.9; HRMS (ESI) calcd for $\mathrm{C}_{11} \mathrm{H}_{10} \mathrm{ClN}_{2} \mathrm{O}[\mathrm{M}+\mathrm{H}]^{+}$221.0482, found 221.0476.

苯甲酰氯-(4-甲基)苯腙 $(4 \mathrm{~m})$ : 白色晶体, $97 \mathrm{mg}$, 产 率 79\%. m.p. $83 \sim 85{ }^{\circ} \mathrm{C}$ (文献值 ${ }^{[2 \mathrm{e}]}: 84 \sim 86{ }^{\circ} \mathrm{C}$ ); ${ }^{1} \mathrm{H}$ $\mathrm{NMR}\left(\mathrm{CDCl}_{3}, 600 \mathrm{MHz}\right) \delta: 7.96(\mathrm{~s}, 1 \mathrm{H}), 7.90 \sim 7.92(\mathrm{~m}$, $2 \mathrm{H}), 7.33 \sim 7.40(\mathrm{~m}, 3 \mathrm{H}), 7.07 \sim 7.11(\mathrm{~m}, 4 \mathrm{H}), 2.30(\mathrm{~s}$, $3 \mathrm{H}) ;{ }^{13} \mathrm{C} \mathrm{NMR}\left(\mathrm{CDCl}_{3}, 150 \mathrm{MHz}\right) \delta: 141.3,134.7,130.6$, $130.0,129.2,128.5,126.5,124.2,113.6,20.8$.

苯甲酰氯-(2-甲基)苯腙(4n): 白色晶体, $85 \mathrm{mg}$, 产 率 69\%. m.p. 66 68 ${ }^{\circ} \mathrm{C}$ (文献值 $\left.{ }^{[6]}: 65 \sim 66{ }^{\circ} \mathrm{C}\right)$; ${ }^{1} \mathrm{H}$ NMR $\left(\mathrm{CDCl}_{3}, 600 \mathrm{MHz}\right) \delta: 7.93 \sim 7.96(\mathrm{~m}, 3 \mathrm{H}), 7.53(\mathrm{~d}, J=8.4$ $\mathrm{Hz} 1 \mathrm{H}), 7.35 \sim 7.42(\mathrm{~m}, 3 \mathrm{H}), 7.22(\mathrm{t}, J=7.8 \mathrm{~Hz}, 1 \mathrm{H}), 7.12$ $(\mathrm{d}, J=7.2 \mathrm{~Hz}, 1 \mathrm{H}), 6.87(\mathrm{t}, J=7.8 \mathrm{~Hz}, 1 \mathrm{H}), 2.30(\mathrm{~s}, 3 \mathrm{H})$; ${ }^{13} \mathrm{C}$ NMR $\left(\mathrm{CDCl}_{3}, 150 \mathrm{MHz}\right) \delta: 141.4,134.6,130.7,129.4$, 128.6, 127.5, 126.6, 125.7, 121.3, 120.9, 113.4, 17.0.

苯甲酰氯-(4-溴)苯腙(4o): 白色晶体, $113 \mathrm{mg}$, 产率 73\%. m.p. $107 \sim 108{ }^{\circ} \mathrm{C}$ (文献值 ${ }^{[2 \mathrm{e}]}$ : $108 \sim 110{ }^{\circ} \mathrm{C}$ ); ${ }^{1} \mathrm{H}$ NMR $\left(\mathrm{CDCl}_{3}, 600 \mathrm{MHz}\right) \delta: 8.02(\mathrm{~s}, 1 \mathrm{H}), 7.90 \sim 7.91(\mathrm{~m}$, 2H), $7.36 \sim 7.42(\mathrm{~m}, 5 \mathrm{H}), 7.05(\mathrm{~d}, J=9.0 \mathrm{~Hz}, 2 \mathrm{H}) ;{ }^{13} \mathrm{C}$ $\mathrm{NMR}\left(\mathrm{CDCl}_{3}, 150 \mathrm{MHz}\right) \delta: 142.6,134.3,132.3,129.6$, 128.6, 126.6, 125.8, 115.2, 113.3.

苯甲酰氯-(3-溴)苯腙 $(4 \mathrm{p})$ : 白色晶体, $121 \mathrm{mg}$, 产率 78\%. m.p. 92 94 ${ }^{\circ} \mathrm{C}$ (文献值 ${ }^{[15]}$ : 90 93 ${ }^{\circ} \mathrm{C}$ ); ${ }^{1} \mathrm{H}$ NMR $\left(\mathrm{CDCl}_{3}, 400 \mathrm{MHz}\right) \delta: 8.03(\mathrm{~s}, 1 \mathrm{H}), 7.90 \sim 7.93(\mathrm{~m}, 2 \mathrm{H})$, $7.38 \sim 7.44(\mathrm{~m}, 4 \mathrm{H}), 7.15(\mathrm{t}, J=8.0 \mathrm{~Hz}, 1 \mathrm{H}), 7.03 \sim 7.06$ $(\mathrm{m}, 2 \mathrm{H}) ;{ }^{13} \mathrm{C} \mathrm{NMR}\left(\mathrm{CDCl}_{3}, 150 \mathrm{MHz}\right) \delta: 144.7,134.3$, $130.8,129.7,128.6,126.7,126.2,124.1,123.5,116.6$, 112.2 .

(4-氯苯基)甲酰氯-(3'-甲基)苯腙(4q)：白色晶体，78 mg, 产率 56\%. m.p. 81 83 ${ }^{\circ} \mathrm{C} ;{ }^{1} \mathrm{H}$ NMR $\left(\mathrm{CDCl}_{3}, 600\right.$ MHz) $\delta$ : $7.98(\mathrm{~s}, 1 \mathrm{H}), 7.83(\mathrm{~d}, J=8.4 \mathrm{~Hz}, 2 \mathrm{H}), 7.36$ (d, $J=$
$8.4 \mathrm{~Hz}, 2 \mathrm{H}), 7.19$ (t, J=7.8 Hz, 1H), $6.98(\mathrm{~s}, 1 \mathrm{H}), 6.96$ (d, $J=7.8 \mathrm{~Hz}, 1 \mathrm{H}), 6.77$ (d, $J=7.2 \mathrm{~Hz}, 1 \mathrm{H}), 2.36(\mathrm{~s}, 3 \mathrm{H}) ;{ }^{13} \mathrm{C}$ NMR $\left(\mathrm{CDCl}_{3}, 150 \mathrm{MHz}\right) \delta: 143.2,139.5,135.2,133.1$, 129.4, 128.7, 127.6, 123.4, 122.4, 114.2, 110.8, 21.8; HRMS (ESI) calcd for $\mathrm{C}_{14} \mathrm{H}_{13} \mathrm{Cl}_{2} \mathrm{~N}_{2}[\mathrm{M}+\mathrm{H}]^{+}$279.0450, found 279.0443 .

(4-氯苯基)甲酰氯-(3'-溴)苯腙(4r): 白色晶体，91 $\mathrm{mg}$, 产率 53\%. m.p. $118 \sim 120{ }^{\circ} \mathrm{C} ;{ }^{1} \mathrm{H} \mathrm{NMR}\left(\mathrm{CDCl}_{3}, 600\right.$ MHz) $\delta: 7.98(\mathrm{~s}, 1 \mathrm{H}), 7.81(\mathrm{~d}, J=9.0 \mathrm{~Hz}, 2 \mathrm{H}), 7.33 \sim 7.36$ $(\mathrm{m}, 3 \mathrm{H}), 7.13(\mathrm{t}, J=8.4 \mathrm{~Hz}, 1 \mathrm{H}), 7.04 \sim 7.05(\mathrm{~m}, 1 \mathrm{H})$, $6.99 \sim 7.01(\mathrm{~m}, 1 \mathrm{H}) ;{ }^{13} \mathrm{C} \mathrm{NMR}\left(\mathrm{CDCl}_{3}, 150 \mathrm{MHz}\right) \delta$ : $144.4,135.6,132.7,130.8,128.8,127.8,124.9,124.3$, 123.5, 116.5, 112.2; GC-MS (EI $70 \mathrm{eV}) \mathrm{m} / z(\%): 344$ (64, $\left.[\mathrm{M}+2]^{+}\right), 342\left(42, \mathrm{M}^{+}\right), 306\left(37,[\mathrm{M}-\mathrm{Cl}-\mathrm{H}]^{+}\right), 227(37$, $\left.[\mathrm{M}-\mathrm{Br}-\mathrm{Cl}-\mathrm{H}]^{+}\right), \quad 169\left(65, \mathrm{C}_{6} \mathrm{H}_{4} \mathrm{BrN}^{+}\right), 90 \quad(100$, $\mathrm{C}_{6} \mathrm{H}_{4} \mathrm{~N}^{+}$).

(4-甲氧基苯基)甲酰氯-(3'-甲基)苯腙(4s): 白色晶 体, $65 \mathrm{mg}$, 产率 47\%. m.p. $119 \sim 121{ }^{\circ} \mathrm{C} ;{ }^{1} \mathrm{H} \mathrm{NMR}$ $\left(\mathrm{CDCl}_{3}, 600 \mathrm{MHz}\right) \delta: 7.83 \sim 7.88(\mathrm{~m}, 3 \mathrm{H}), 7.10(\mathrm{~d}, J=8.4$ $\mathrm{Hz}, 2 \mathrm{H}), 7.06$ (d, $J=8.4 \mathrm{~Hz}, 2 \mathrm{H}), 6.92$ (d, $J=9.0 \mathrm{~Hz}, 2 \mathrm{H})$, 3.85 (s, 3H), $2.30(\mathrm{~s}, 3 \mathrm{H}) ;{ }^{13} \mathrm{C} \mathrm{NMR}\left(\mathrm{CDCl}_{3}, 150 \mathrm{MHz}\right) \delta$ : $160.9,137.5,130.7,129.7,128.7,128.2,117.7,114.6$, 114.0, 55.6, 20.5; HRMS (ESI) calcd for $\mathrm{C}_{15} \mathrm{H}_{15} \mathrm{ClN}_{2} \mathrm{ONa}$ $[\mathrm{M}+\mathrm{Na}]^{+}$297.0765, found 297.0768 .

(4-甲氧基苯基)甲酰氯-(3'-溴)苯腙(4t): 白色晶体, $70 \mathrm{mg}$, 产率 41\%. m.p. 125 127 ${ }^{\circ} \mathrm{C} ;{ }^{1} \mathrm{H} \mathrm{NMR}\left(\mathrm{CDCl}_{3}\right.$, $400 \mathrm{MHz}) \delta: 7.93(\mathrm{~s}, 1 \mathrm{H}), 7.85(\mathrm{~d}, J=9.2 \mathrm{~Hz}, 2 \mathrm{H}), 7.36(\mathrm{t}$, $J=1.6 \mathrm{~Hz}, 1 \mathrm{H}), 7.14(\mathrm{t}, J=8.0 \mathrm{~Hz}, 1 \mathrm{H}), 7.00 \sim 7.04(\mathrm{~m}$, $2 \mathrm{H}), 6.93(\mathrm{~d}, J=8.8 \mathrm{~Hz}, 2 \mathrm{H}), 3.85(\mathrm{~s}, 3 \mathrm{H}) ;{ }^{13} \mathrm{C} \mathrm{NMR}$ $\left(\mathrm{CDCl}_{3}, 150 \mathrm{MHz}\right) \delta: 161.0,145.0,130.7,128.2,126.9$, 126.2, 123.8, 123.5, 116.4, 114.0, 112.1, 55.6; HRMS (ESI) calcd for $\mathrm{C}_{14} \mathrm{H}_{12} \mathrm{BrClN}_{2} \mathrm{ONa}[\mathrm{M}+\mathrm{Na}]^{+}$360.9714, found 360.9710 .

肉桂基甲酰氯苯腙 $(4 \mathbf{u})$ : 黄色晶体, $95 \mathrm{mg}$, 产率 74\%. m.p. $151 \sim 153{ }^{\circ} \mathrm{C}$ (文献值 ${ }^{[6]}: 149 \sim 151{ }^{\circ} \mathrm{C}$ ); ${ }^{1} \mathrm{H}$ NMR $\left(\mathrm{CDCl}_{3}, 400 \mathrm{MHz}\right) \delta: 8.04(\mathrm{~s}, 1 \mathrm{H}), 7.50$ (d, $J=7.6$ $\mathrm{Hz}, 2 \mathrm{H}), 7.28 \sim 7.38(\mathrm{~m}, 5 \mathrm{H}), 7.08 \sim 7.14(\mathrm{~m}, 3 \mathrm{H}), 6.92 \sim$ $7.00(\mathrm{~m}, 2 \mathrm{H}) ;{ }^{13} \mathrm{C} \mathrm{NMR}\left(\mathrm{CDCl}_{3}, 150 \mathrm{MHz}\right) \delta: 143.1$, $136.2,133.5,129.5,129.0,128.6,127.1,126.2,123.5$, 121.5, 113.6; HRMS (ESI) calcd for $\mathrm{C}_{15} \mathrm{H}_{14} \mathrm{ClN}_{2}[\mathrm{M}+\mathrm{H}]^{+}$ 257.0840 , found 257.0834 .

苯乙基甲酰氯苯腙 ${ }^{[6]}(\mathbf{4 v})$ : 无色油状液体, $99 \mathrm{mg}$, 产率 76\%. ${ }^{1} \mathrm{H} \mathrm{NMR}\left(\mathrm{CDCl}_{3}, 600 \mathrm{MHz}\right) \delta: 7.61(\mathrm{~s}, 1 \mathrm{H})$, $7.29(\mathrm{t}, J=7.2 \mathrm{~Hz}, 2 \mathrm{H}), 7.19 \sim 7.26(\mathrm{~m}, 5 \mathrm{H}), 7.01(\mathrm{~d}, J=$ $7.8 \mathrm{~Hz}, 2 \mathrm{H}), 6.88(\mathrm{t}, J=7.2 \mathrm{~Hz}, 1 \mathrm{H}), 3.04(\mathrm{t}, J=7.2 \mathrm{~Hz}$, 
2H), $2.91 \sim 2.93(\mathrm{~m}, 2 \mathrm{H}) ;{ }^{13} \mathrm{C} \mathrm{NMR}\left(\mathrm{CDCl}_{3}, 150 \mathrm{MHz}\right) \delta$ : $143.9,140.4,129.4,128.6,128.6,126.6,126.4,120.8$, 113.3, 40.7, 33.1.

环戊基甲酰氯苯腙 $(4 \mathrm{w})$ : 淡黄色油状液体， $64 \mathrm{mg}$, 产率 58\%. ${ }^{1} \mathrm{H}$ NMR $\left(\mathrm{CDCl}_{3}, 600 \mathrm{MHz}\right) \delta: 7.60(\mathrm{~s}, 1 \mathrm{H})$, 7.25 (t, $J=8.4 \mathrm{~Hz}, 2 \mathrm{H}), 7.03(\mathrm{~d}, J=7.8 \mathrm{~Hz}, 2 \mathrm{H}), 6.87$ (t, $J=7.8 \mathrm{~Hz}, 1 \mathrm{H}), 3.02 \sim 3.08(\mathrm{~m}, 1 \mathrm{H}), 1.85 \sim 1.96(\mathrm{~m}, 4 \mathrm{H})$, $1.72 \sim 1.79(\mathrm{~m}, 2 \mathrm{H}), 1.59 \sim 1.66(\mathrm{~m}, 2 \mathrm{H}) ;{ }^{13} \mathrm{C}$ NMR $\left(\mathrm{CDCl}_{3}, 150 \mathrm{MHz}\right) \delta: 144.2,131.6,129.4,120.6,113.2$, 48.5, 30.9, 25.4 .

环己基甲酰氯苯腙 ${ }^{[2 f]}(\mathbf{4 x})$ : 淡黄色油状液体, $75 \mathrm{mg}$, 产率 64\%. ${ }^{1} \mathrm{H}$ NMR $\left(\mathrm{CDCl}_{3}, 600 \mathrm{MHz}\right) \delta: 7.60(\mathrm{~s}, 1 \mathrm{H})$, $7.24(\mathrm{t}, J=9.0 \mathrm{~Hz}, 2 \mathrm{H}), 7.02(\mathrm{~d}, J=7.2 \mathrm{~Hz}, 2 \mathrm{H}), 6.86(\mathrm{t}$, $J=7.2 \mathrm{~Hz}, 1 \mathrm{H}), 2.48 \sim 2.53(\mathrm{~m}, 1 \mathrm{H}), 2.02(\mathrm{~d}, J=13.2 \mathrm{~Hz}$, $2 \mathrm{H}), 1.80 \sim 1.83(\mathrm{~m}, 2 \mathrm{H}), 1.67 \sim 1.70(\mathrm{~m}, 1 \mathrm{H}), 1.48 \sim 1.54$ $(\mathrm{m}, 2 \mathrm{H}), 1.19 \sim 1.36(\mathrm{~m}, 3 \mathrm{H}) ;{ }^{13} \mathrm{C}$ NMR $\left(\mathrm{CDCl}_{3}, 150\right.$ MHz) $\delta: 144.2,132.1,129.4,120.5,113.2,47.2,30.9$, 26.1, 25.8.

\section{2 .2 化合物 6 的合成}

在 $25 \mathrm{~mL}$ 的圆底烧瓶中, 依次加入三分之一的 $\operatorname{TCCA}(0.2 \mathrm{mmol}, 47 \mathrm{mg}) 、 N, N$-二甲基甲酰胺 $(3 \mathrm{~mL})$ 、三 氟乙醛苯腙 $(5 \mathbf{a})(0.5 \mathrm{mmol}, 94 \mathrm{mg})$, 室温下搅拌上述反 应混合物 $5 \mathrm{~min}$ 后, 再加入剩余的 TCCA, 继续搅拌. TLC 监测 $15 \mathrm{~min}$ 反应完全. 此时, 向反应体系中加水 $(10 \mathrm{~mL})$ 淬灭反应, 用乙酸乙酯萃取 $(10 \mathrm{~mL} \times 3)$, 有机相 依次用蒸馏水洗涤 $(10 \mathrm{~mL} \times 3)$, 饱和氯化钠水溶液 $(10$ $\mathrm{mL}$ )洗涤, 无水硫酸美干燥, 抽滤, 减压除去溶剂. 残留 物进行柱层析 $[V$ (石油醚) $: V$ (乙酸乙酯 $)=20: 1]$ 分离得 三氟乙酰氯苯腙 ${ }^{[10]}(\mathbf{6 a})$. 淡黄色油状液体, $94 \mathrm{mg}$, 产率 $84 \% .{ }^{1} \mathrm{H} \mathrm{NMR}\left(\mathrm{CDCl}_{3}, 600 \mathrm{MHz}\right) \delta: 8.02(\mathrm{~s}, 1 \mathrm{H}), 7.32(\mathrm{t}$, $J=7.8 \mathrm{~Hz}, 2 \mathrm{H}), 7.13$ (d, $J=7.8 \mathrm{~Hz}, 2 \mathrm{H}), 7.03$ (t, $J=7.2$ $\mathrm{Hz}, 1 \mathrm{H}) ;{ }^{13} \mathrm{C}$ NMR $\left(\mathrm{CDCl}_{3}, 150 \mathrm{MHz}\right) \delta: 141.5,129.7$, $123.2,118.5\left(\mathrm{q}, J_{\mathrm{C}-\mathrm{F}}=268.5 \mathrm{~Hz}\right), 114.3,104.0\left(\mathrm{q}, J_{\mathrm{C}-\mathrm{F}}=\right.$ $43.5 \mathrm{~Hz}) ;{ }^{19} \mathrm{~F}$ NMR $\left(376 \mathrm{MHz}, \mathrm{CDCl}_{3}\right) \delta: 68.2$.

化合物 $\mathbf{6 b} \sim \mathbf{6 h}$ 的合成方法同 $\mathbf{6 a}$.

三氟乙酰氯-(4-甲基)苯腙(6b): 淡红色油状液体, $84 \mathrm{mg}$, 产率 71\%. ${ }^{1} \mathrm{H} \mathrm{NMR}\left(\mathrm{CDCl}_{3}, 600 \mathrm{MHz}\right) \delta: 7.95$ (s, 1H), 7.12 (d, $J=7.8 \mathrm{~Hz}, 2 \mathrm{H}), 7.03$ (d, $J=8.4 \mathrm{~Hz}, 2 \mathrm{H}), 2.31$ $(\mathrm{s}, 3 \mathrm{H}) ;{ }^{13} \mathrm{C} \mathrm{NMR}\left(\mathrm{CDCl}_{3}, 150 \mathrm{MHz}\right) \delta: 139.5,132.6$, $130.1,118.7\left(\mathrm{q}, J_{\mathrm{C}^{-} \mathrm{F}}=270 \mathrm{~Hz}\right), 114.1,110.9\left(\mathrm{q}, J_{\mathrm{C}^{-} \mathrm{F}}=\right.$ $43.5 \mathrm{~Hz}), 20.8 ;{ }^{19} \mathrm{~F}$ NMR (376 MHz, $\left.\mathrm{CDCl}_{3}\right) \delta: 68.1$; GC-MS (EI $70 \mathrm{eV}) \mathrm{m} / z(\%): 238\left(28,[\mathrm{M}+2]^{+}\right), 236(92$, $\left.\mathrm{M}^{+}\right), 106\left(100, \mathrm{C}_{6} \mathrm{H}_{4} \mathrm{CH}_{3} \mathrm{NH}^{+}\right), 91\left(7, \mathrm{C}_{6} \mathrm{H}_{4} \mathrm{CH}_{3}{ }^{+}\right), 69(6$, $\mathrm{CF}_{3}{ }^{+}$).

三氟乙酰氯-(4-甲氧基)苯腙 ${ }^{[16]}(\mathbf{6 c})$ : 淡红色油状液
体, $82 \mathrm{mg}$, 产率 $65 \% .{ }^{1} \mathrm{H} \mathrm{NMR}\left(\mathrm{CDCl}_{3}, 600 \mathrm{MHz}\right) \delta: 7.90$ (s, $1 \mathrm{H}), 7.06 \sim 7.08(\mathrm{~m}, 2 \mathrm{H}), 6.86 \sim 6.89(\mathrm{~m}, 2 \mathrm{H}), 3.79$ (s, $3 \mathrm{H}) ;{ }^{13} \mathrm{C} \mathrm{NMR}\left(\mathrm{CDCl}_{3}, 150 \mathrm{MHz}\right) \delta: 155.8,135.6,118.7$ $\left(\mathrm{q}, J_{\mathrm{C}-\mathrm{F}}=268.5 \mathrm{~Hz}\right), 115.5,114.9,110.6\left(\mathrm{q}, J_{\mathrm{C}-\mathrm{F}}=43.5\right.$ $\mathrm{Hz}), 55.8 ;{ }^{19} \mathrm{~F}$ NMR $\left(376 \mathrm{MHz}, \mathrm{CDCl}_{3}\right) \delta: 68.0$.

三氟乙酰氯-(4-溴) 苯腙 ${ }^{[16]}(\mathbf{6 d})$ : 淡黄色油状液体, $127 \mathrm{mg}$, 产率 84\%. ${ }^{1} \mathrm{H}$ NMR $\left(\mathrm{CDCl}_{3}, 600 \mathrm{MHz}\right) \delta: 7.99$ (s, 1H), $7.42 \sim 7.44(\mathrm{~m}, 2 \mathrm{H}), 7.01 \sim 7.04(\mathrm{~m}, 2 \mathrm{H}) ;{ }^{13} \mathrm{C}$ $\operatorname{NMR}\left(\mathrm{CDCl}_{3}, 150 \mathrm{MHz}\right) \delta: 140.9,132.6,118.5\left(\mathrm{q}, J_{\mathrm{C}^{-} \mathrm{F}}=\right.$ $270.0 \mathrm{~Hz}), 115.8,115.4,112.6\left(\mathrm{q}, J_{\mathrm{C}-\mathrm{F}}=43.5 \mathrm{~Hz}\right) ;{ }^{19} \mathrm{~F}$ NMR (376 MHz, $\left.\mathrm{CDCl}_{3}\right) \delta: 68.3$.

三氟乙酰氯-(3-溴)苯腙 $(6 e)$ : 淡红色油状液体, 116 $\mathrm{mg}$, 产率 77\%. ${ }^{1} \mathrm{H} \mathrm{NMR}\left(\mathrm{CDCl}_{3}, 600 \mathrm{MHz}\right) \delta: 7.97(\mathrm{~s}$, $1 \mathrm{H}), 7.32$ (t, $J=1.8 \mathrm{~Hz}, 1 \mathrm{H}), 7.13 \sim 7.18(\mathrm{~m}, 2 \mathrm{H}), 7.03$ (dt, $J=7.8,1.8 \mathrm{~Hz}, 1 \mathrm{H}) ;{ }^{13} \mathrm{C}$ NMR $\left(\mathrm{CDCl}_{3}, 150 \mathrm{~Hz}\right) \delta: 142.8$, $130.8,125.8,123.3,118.3\left(\mathrm{q}, J_{\mathrm{C}-\mathrm{F}}=270.0 \mathrm{~Hz}\right), 117.0$, $112.9\left(\mathrm{q}, J_{\mathrm{C}-\mathrm{F}}=42.0 \mathrm{~Hz}\right), 112.6 ;{ }^{19} \mathrm{~F}$ NMR $(376 \mathrm{MHz}$, $\mathrm{CDCl}_{3}$ ) $\delta$ : 68.4; GC-MS (EI $\left.70 \mathrm{eV}\right) \mathrm{m} / z$ (\%): 304 (24, $\left.[\mathrm{M}+4]^{+}\right), 302\left(100,[\mathrm{M}+2]^{+}\right), 300\left(77, \mathrm{M}^{+}\right), 186(24$, $\left.[\mathrm{M}-\mathrm{Br}-\mathrm{Cl}]^{+}\right), \quad 170 \quad\left(30, \mathrm{C}_{6} \mathrm{H}_{4} \mathrm{BrNH}^{+}\right), 91 \quad(60$, $\mathrm{C}_{6} \mathrm{H}_{4} \mathrm{NH}^{+}$).

三氟乙酰氯-(2-溴)苯腙(6f): 淡黄色油状液体, 110 $\mathrm{mg}$, 产率 73\%. ${ }^{1} \mathrm{H}$ NMR $\left(\mathrm{CDCl}_{3}, 600 \mathrm{MHz}\right) \delta: 8.55(\mathrm{~s}$, $1 \mathrm{H}), 7.48(\mathrm{td}, J=8.4,1.2 \mathrm{~Hz}, 2 \mathrm{H}), 7.30$ (t, $J=7.8 \mathrm{~Hz}, 1 \mathrm{H})$, $6.89(\mathrm{td}, J=7.8,1.8 \mathrm{~Hz}, 1 \mathrm{H}) ;{ }^{13} \mathrm{C} \mathrm{NMR}\left(\mathrm{CDCl}_{3}, 150 \mathrm{MHz}\right)$ $\delta: 138.8,132.7,129.0,123.7,118.5\left(\mathrm{q}, J_{\mathrm{C}-\mathrm{F}}=271.5 \mathrm{~Hz}\right)$, $115.7,114.2\left(\mathrm{q}, J_{\mathrm{C}-\mathrm{F}}=43.5 \mathrm{~Hz}\right), 108.5 ;{ }^{19} \mathrm{~F}$ NMR $(376$ $\mathrm{MHz}, \mathrm{CDCl}_{3}$ ) $\delta$ : 68.4; GC-MS (EI $70 \mathrm{eV}$ ) m/z (\%): 304 $\left(25,[\mathrm{M}+4]^{+}\right), 302\left(100,[\mathrm{M}+2]^{+}\right), 300\left(85, \mathrm{M}^{+}\right), 186(9$, $\left.[\mathrm{M}-\mathrm{Br}-\mathrm{Cl}]^{+}\right), \quad 170 \quad\left(44, \mathrm{C}_{6} \mathrm{H}_{4} \mathrm{BrNH}^{+}\right), 91 \quad(71$, $\mathrm{C}_{6} \mathrm{H}_{4} \mathrm{NH}^{+}$).

三氟乙酰氯-(4-氟)苯腙(6g): 淡红色油状液体, 103 $\mathrm{mg}$, 产率 86\%. ${ }^{1} \mathrm{H}$ NMR $\left(\mathrm{CDCl}_{3}, 600 \mathrm{MHz}\right) \delta: 7.96(\mathrm{~s}$, $1 \mathrm{H}), 7.08 \sim 7.11(\mathrm{~m}, 2 \mathrm{H}), 7.01 \sim 7.05(\mathrm{~m}, 2 \mathrm{H}) ;{ }^{13} \mathrm{C}$ NMR $\left(\mathrm{CDCl}_{3}, 150 \mathrm{MHz}\right) \delta: 158.9\left(\mathrm{~d}, J_{\mathrm{C}-\mathrm{F}}=240.0 \mathrm{~Hz}\right), 138.0(\mathrm{~d}$, $\left.J_{\mathrm{C}-\mathrm{F}}=1.5 \mathrm{~Hz}\right), 118.6\left(\mathrm{q}, J_{\mathrm{C}-\mathrm{F}}=270.0 \mathrm{~Hz}\right), 116.3\left(\mathrm{~d}, J_{\mathrm{C}-\mathrm{F}}=\right.$ $22.5 \mathrm{~Hz}), 115.4\left(\mathrm{~d}, J_{\mathrm{C}-\mathrm{F}}=7.5 \mathrm{~Hz}\right), 111.8\left(\mathrm{q}, J_{\mathrm{C}-\mathrm{F}}=46.5\right.$ $\mathrm{Hz}) ;{ }^{19} \mathrm{~F}$ NMR $\left(376 \mathrm{MHz}, \mathrm{CDCl}_{3}\right) \delta: 68.3,121.3$; GC-MS (EI $70 \mathrm{eV}) \mathrm{m} / z(\%): 242\left(25,[\mathrm{M}+2]^{+}\right), 240\left(67, \mathrm{M}^{+}\right), 185$ $\left(6,[\mathrm{M}-\mathrm{Br}-\mathrm{Cl}-\mathrm{H}]^{+}\right), 110\left(100, \mathrm{C}_{6} \mathrm{H}_{4} \mathrm{FNH}^{+}\right), 95$ (7, $\left.\mathrm{C}_{6} \mathrm{H}_{4} \mathrm{~F}^{+}\right), 69\left(7, \mathrm{CF}_{3}^{+}\right)$.

三氟乙酰氯-(4-硝基)苯腙(6h): 淡黄色固体，110 $\mathrm{mg}$, 产率 82\%. m.p. $131 \sim 133{ }^{\circ} \mathrm{C} ;{ }^{1} \mathrm{H}$ NMR $\left(\mathrm{CDCl}_{3}, 600\right.$ MHz) $\delta: 8.44(\mathrm{~s}, 1 \mathrm{H}), 8.22 \sim 8.25(\mathrm{~m}, 2 \mathrm{H}), 7.25 \sim 7.27(\mathrm{~m}$, $2 \mathrm{H}) ;{ }^{13} \mathrm{C} \mathrm{NMR}\left(\mathrm{CDCl}_{3}, 150 \mathrm{MHz}\right) \delta: 146.9,142.9,126.0$, 
$118.2\left(\mathrm{q}, J_{\mathrm{C}-\mathrm{F}}=270.0 \mathrm{~Hz}\right), 115.8\left(\mathrm{q}, J_{\mathrm{C}-\mathrm{F}}=43.5 \mathrm{~Hz}\right)$, 113.9; ${ }^{19} \mathrm{~F}$ NMR $\left(376 \mathrm{MHz}, \mathrm{CDCl}_{3}\right) \delta$ : 68.7; HRMS (ESI) calcd for $\mathrm{C}_{8} \mathrm{H}_{5} \mathrm{ClF}_{3} \mathrm{~N}_{3} \mathrm{O}_{2} \mathrm{Na}[\mathrm{M}+\mathrm{Na}]^{+}$289.9915, found 289.9915.

辅助材料(Supporting Information) 所合成产物的 ${ }^{1} \mathrm{H}$ NMR 和 ${ }^{13} \mathrm{C}$ NMR 谱图. 这些材料可以免费从本刊网站 (http://sioc-journal.cn/)上下载.

\section{References}

[1] (a) Singh, M. S.; Chowdhury, S.; Koley, S. Tetrahedron 2016, 72, 1603.

(b) Hashimoto, T.; Maruoka, K. Chem. Rev. 2015, 115, 5366.

(c) Shawali, A. S. Chem. Rev. 1993, 93, 2731.

(d) Hu, X.; Liu, B.; Liu, H.; Li, X. Chin. J. Org. Chem. 2013, 33, 1583 (in Chinese)

(胡小莲, 刘涁, 刘浩冲, 李篵芳, 有机化学, 2013, 33, 1583.)

[2] For selected recent artiles see:

(a) Sharma, P.; Bhat, S. V.; Prabhath, M. R. R; Molino, A.; Nauha, E.; Wilson, D. J. D.; Moses, J. E. Org. Lett. 2018, 20, 4263.

(b) Guo, C.-X.; Zhang, W.-Z.; Zhang, N.; Lu, X.-B. J. Org. Chem. 2017, 82, 7637.

(c) Liu, H.; Jia, H.; Wang, B.; Xiao, Y.; Guo, H. Org. Lett. 2017, 19, 4714 .

(d) Tsai, S.-E.; Yen, W.-P.; Li, Y.-T.; Hu, Y.-T.; Tseng, C.-C.; Wong, F. F. Asian J. Org. Chem. 2017, 6, 1470.

(e) Wang, G.; Liu, X.; Huang, T.; Kuang, Y.; Lin, L.; Feng, X. Org. Lett. 2013, 15, 76.

(f) Fuchi, N.; Doi, T.; Takahashi, T. Chem. Lett. 2005, 34, 438.

[3] (a) Zhao, H.-W.; Zhao, Y.-D.; Liu, Y.-Y.; Zhao, L.-J.; Song, X.-Q.; Chen, X.-Q.; Pang, H.-L.; Du, J.; Feng, N.-N. RSC Adv. 2017, 7, 55106.

(b) Garve, L. K. B.; Petzold, M.; Jones, P. G.; Werz, D. B. Org. Lett. 2016, 18,564

[4] Guo, Z.; Jia, H.; Liu, H.; Wang, Q.; Huang, J.; Guo, H. Org. Lett.
2018, 20, 2939.

[5] Ulrich, H. The Chemistry of Imidoyl Halides, Plenum Press, New York, 1968; Chapter 7, p. 173.

[6] Patel, H. V.; Vyas, K. A.; Pandey, S. P.; Fernandes, P. S. Tetrahedron 1996, 52, 661.

[7] (a) Pinto, G.; Rohrig, B. J. Chem. Educ. 2003, 80, 41.

(b) Tilstam, U.; Weinmann, H. Org. Process Res. Dev. 2002, 6, 384.

[8] (a) Su, Y.; Shi, Y.; Chang, B.; Wu, L.; Chong, S.; Zhang, W.; Huang, D.; Wang, K.-H.; Hu, Y. Chin. J. Org. Chem. 2018, 38, 1454 (in Chinese).

(苏瀛鹏，石娅，常兵兵，吴丽丽，种思颖，张为钢，黄丹凤，王 克虎, 胡雨来, 有机化学, 2018, 38, 1454.)

(b) Motati, D. R.; Uredi, D.; Watkins, E. B. Chem. Sci. 2018, 9, 1782 .

(c) Combe, S. H.; Hosseini, A.; Parra, A.; Schreiner, P. R. J. Org. Chem. 2017, 82, 2407.

(d) Zhao, N.; Xuan, S.; Fronczek, F. R.; Smith, K. M.; Vicente, M. G. H. J. Org. Chem. 2015, 80, 8377.

(e) D’Oyley, J. M.; Aliev, A. E.; Sheppard, T. D. Angew. Chem. Int. Ed. 2014, 53, 10747.

(f) Vo, T. T.; Zhang, J.; Parrish, D. A.; Twamley, B.; Shreeve, J. M. J. Am. Chem. Soc. 2013, 135, 11787.

[9] (a) Gaspa, S.; Porcheddu, A.; Luca, L. D. Adv. Synth. Catal. 2016, $358,154$.

(b) Gaspa, S.; Porcheddu, A.; Luca, L. D. Org. Lett. 2015, 17, 3666. (c) Jing, Y.; Daniliuc, C. G.; Studer, A. Org. Lett. 2014, 16, 4932.

[10] Tanaka, K.; Maeno, S.; Mitsuhashi, K. J. Heterocycl. Chem. 1985, 22,565 .

[11] Corey, E. J.; Kim, C. U. J. Am. Chem. Soc. 1972, 94, 7586.

[12] Yatham, V. R.; Harnying, W.; Kootz, D.; Neudorfl, J.-M.; Schlorer, N. E.; Berkessel, A. J. Am. Chem. Soc. 2016, 138, 2670.

[13] Wojciechowska, A.; Jasinski, M.; Kaszynski, P. Tetrahedron 2015, $71,2349$.

[14] Giustiniano, M.; Meneghetti, F.; Mercalli, V.; Varese, M.; Giustiniano, F.; Novellino, E.; Tron, G. C. Org. Lett. 2014, 16, 5332.

[15] Palazzino, G.; Cecchi, H.; Melani, F.; Colotta, V.; Filacchioni, G.; Martini, C.; Lucacchini, A. J. Med. Chem. 1987, 30, 1737.

[16] Ellsworth, B. A.; Shi, J.; Ewing, W. R.; Jurica, E. A.; Hernandez, A. S.; Wu, X. US 0142139, 2014 [Chem. Abstr. 2014, 161, 830876].

(Li, L.; Fan, Y.) 